\title{
From Stirring to Mixing in a Stratified Ocean
}

\author{
Peter Müller \\ University of Hawaii • Honolulu, Hawaii USA
}

\author{
Chris Garrett \\ University of Victoria - Victoria, British Columbia Canada
}

Over fifty years ago, Carl Eckart described the sequence of processes as a passive scalar is stirred and mixed in a turbulent flow (Eckart, 1948). At first, during the stirring phase, the variance of the scalar gradient is greatly increased, but later, during the mixing phase, the gradients become sufficiently sharp that molecular diffusion becomes important and the gradient variance rapidly decreases. The process is of great practical importance in many engineering situations as well as being familiar from adding cream to coffee.

The interplay of stirring and mixing is also important in the ocean for temperature, salinity, chlorophyll and other naturally occurring tracers as well as for introduced material. The situation is complicated by the numerous physical processes that can cause stirring and by the interplay of processes that act along isopycnals (surfaces of constant mean density, where "mean" requires careful definition) with diapycnal processes acting across the density surfaces.

The Twelfth 'Aha Huliko'a Hawaiian Winter Workshop was convened from 15 to 19 January 2001 to review this theme, with the support of the Office of Naval Research, the Joint Institute for Marine and Atmospheric Research, and the participation of 25 invited speakers. The general topics included in presentations and discussions were (1) mesoscale eddy parameterization, (2) general approaches to stirring, including the application of ideas from dynamical systems theory, (3) inertial instability, submesoscale motions and vortical motions, (4) the interplay of isopycnal and diapycnal processes, (5) processes in the surface mixed layer, (6) the stirring and mixing of nonconservative, particularly biological, tracers, (7) mixing efficiency, i.e., the fraction of energy lost from the mean flow which produces a vertical buoyancy flux, and (8) differential mixing of heat and salt in doubly-stable environments as well as those which permit double diffusion. Before summarizing the discussions on these topics, we first review the general approach to parameterizing unresolved processes.

\section{Parameterizing Fluxes by Unresolved Processes}

Ocean general circulation models used in studies of climate, biological productivity and waste disposal lack the resolution to treat small-scale processes explicitly and so must parameterize their effects in terms of the average properties that are resolved. Any model that aspires to predictive capability requires an understanding of the dynamical processes responsible for the transports to which the model output is sensitive. The parameterizations must then be specified as formulae rather than just numerical values.

This need to parameterize unresolved processes is not idiosyncratic to oceanic general circulation models but generic to models in many fields. In the atmosphere, for example, one thinks of clouds. The need to understand these is readily accepted.

In the ocean one might consider the example of diapycnal mixing by internal wave breaking. If this is to be characterized not by a number but by a formula then the origin of the wave field becomes important. Vastly different feedback loops can be obtained in climate studies depending on whether the intensity of the wave field and hence diapycnal mixing is determined by the ocean circulation, cascading energy via eddies to the waves, or by the atmospheric wind field generating waves at the sea surface, or by the surface tide generating waves at the sea floor.

The simplest view of the flux produced by unresolved processes is that it is down the mean gradient, i.e., at right angles to the surfaces of constant mean values of the property in question, and may be described in terms of an "eddy diffusivity" that is larger than the molecular diffusivity. This view derives from the classi- 


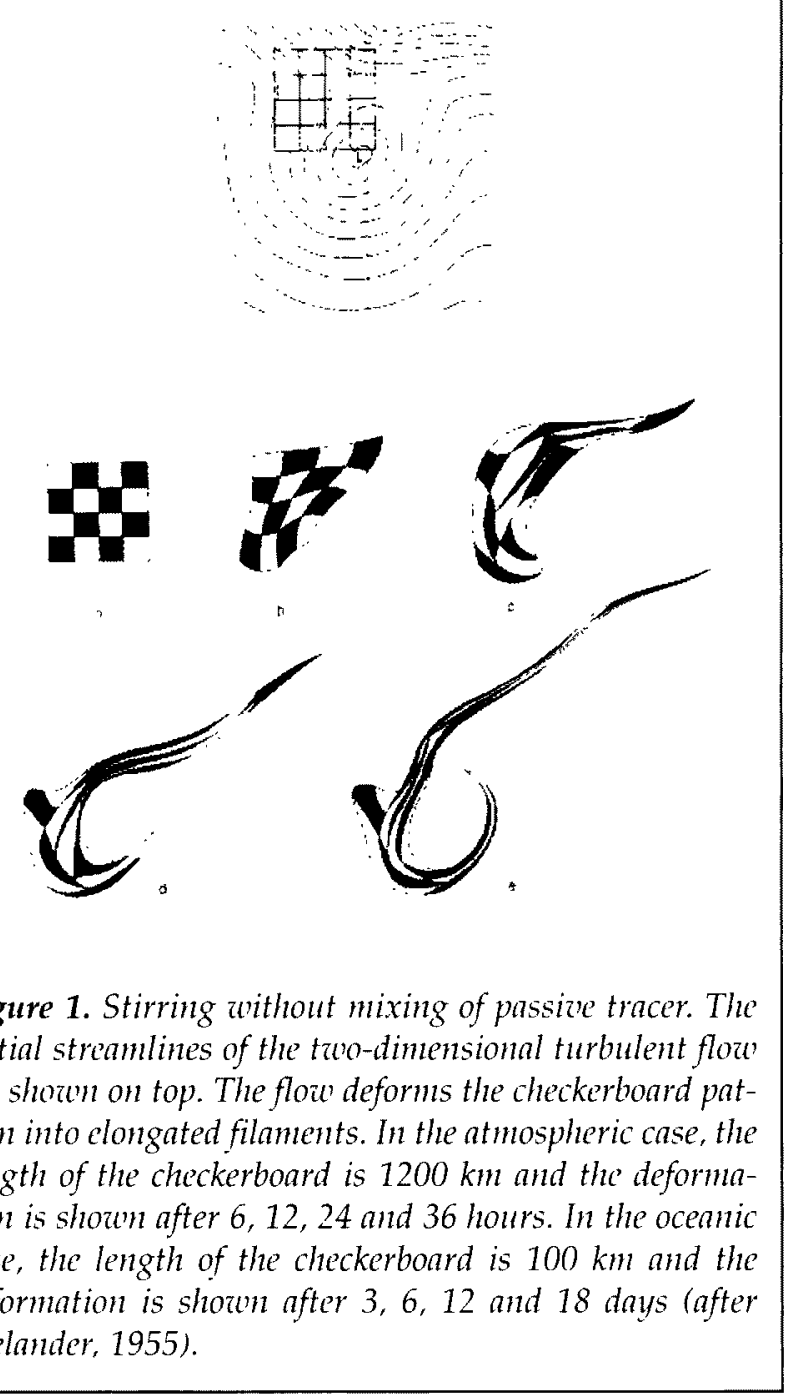

cal mixing length argument. There can, however, be an additional flux parallel to the surfaces of constant mean value. A simple example would be for surface water waves in a temperature-stratified ocean with, say, the temperature increasing upwards. Water particles have circular orbits (in deep water), moving in the direction of wave propagation at the top of the orbit and in the opposite direction at the bottom. Thus the water moving forward is cold, having come from below, and the water moving back is warm, having come from above. This results in an apparent heat flux, called the "skew" flux, normal to the mean temperature gradient and in the direction opposite to that of the wave propagation in this case.

This concept is embodied in a general mathematical approach that uses a tensor to relate the eddy flux of a scalar to the local gradient of the mean concentration of the scalar. The symmetric part of the tensor is diagonalizable and may be thought of as anisotropic diffusion. The general assumption is that two principal directions are along the "neutral" surfaces of constant mean potential density, with very much greater diffu- sivity in these directions than in the direction normal to the surfaces. The isopycnal diffusion along the neutral surfaces is largely associated with mesoscale eddies, whereas the diapycnal mixing across them is caused by breaking internal waves and, in some locations, double-diffusive processes.

The antisymmetric part of the tensor describes a skew flux normal to the mean gradient of the scalar. Part of this flux is non-divergent and does not affect the mean scalar concentration. The remaining, divergent part may be expressed as simple advection of the scalar by an additional mean flow, the "skew velocity," over and above that which would be recorded by current meters at a fixed location. To some extent the skew flux can be thought of as representing an eddy flux of thickness, or spacing between neighboring isopycnals, but it is actually a more subtle representation, in a coordinate system using $z$ as the vertical coordinate, of a flux that would appear in a coordinate system using the potential density instead.

This formulation is purely kinematic. Specific numerical values for the diffusivities and skew velocities could be obtained from suitable measurements in the present ocean, though we have stressed the need for formulae rather than just values appropriate to the present ocean.

\section{Mesoscale Eddies}

So-called mesoscale eddies, with a spatial scale of the order of $100 \mathrm{~km}$ and a time scale of the order of a month, are a dominant feature of ocean variability. They are dynamically analogous to synoptic-scale eddies in the atmosphere. The deformation of an air layer by such eddies is depicted in Pierre Welander's (1955) now famous checkerboard figure, here reproduced as Figure 1. The figure also depicts the stirring along oceanic isopycnals if time and space are appropriately rescaled.

Determining the magnitudes of the isopycnal diffusivity and skew velocity associated with oceanic mesoscale eddies requires an understanding derived from a combination of general principles, studies of dynamical processes such as baroclinic instability, and numerical models that resolve the eddies in various idealized situations. There are no simple answers. To some extent the eddies serve to release available potential energy from the mean state, as in the popular scheme of Gent and McWilliams (1990), driving the ocean toward a state of rest with flat isopycnals. Other schemes assume that eddies mix potential vorticity; driving the ocean toward a state of uniform potential vorticity on isopycnal surfaces. Idealized eddyresolving models, on the other hand, show that eddies drive anticyclonic circulations around seamounts with neither flat isopycnals nor homogeneous potential vorticity distribution. Moreover, idealized eddy-resolving runs have mostly been used to produce numerical values for the free parameters in the various parameterization 

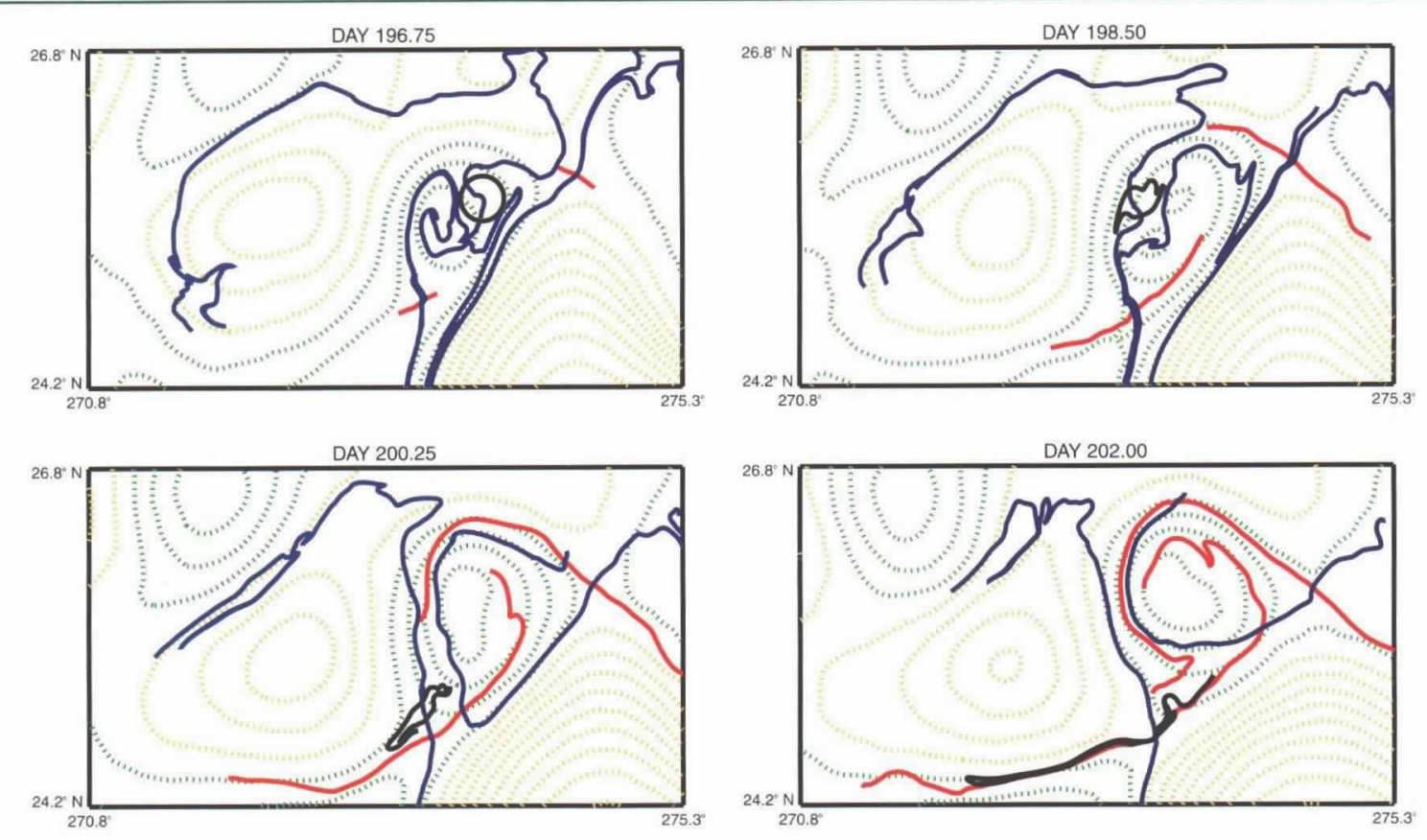

Figure 2. Evolution of a blob of tracer in a simulation of the surface currents in the Gulf of Mexico. The Loop Current is located in the lower right corner and a cyclone to the northwest. Height anomalies are shown as dashed lines. The red and blue lines are inflowing and outflowing manifolds. There are two hyperbolic regions associated with the cyclone and the Loop Current. On day 196.75 a circular blob (black) straddles an inflowing manifold (blue). This manifold delineates which portion of the blob will travel west into the central Gulf and which portion will travel east through the Straits of Florida. The blob is stirred with modest deformation for the next 3.5 days as it rotates around the cyclone. On day 200.25, however, the blob approaches the hyperbolic point created by the intersecting inflowing (blue) and outflowing (red) manifolds to the southwest of the cyclone. Here it begins to show strong compression along the inflowing manifold direction and strong distortion along the outflowing manifold direction. Day 202.00 shows the fate of the blob when it reaches this intersection. It has collapsed along the outflowing manifold. (Courtesy A.D. Kirwan, L. Kantha, and M. Toner.)

schemes, rather than the formula in terms of resolved variables, which we really need for predictive models.

The extant parameterizations of mesoscale eddies treat the stirring effect of the eddies. One unanswered question is whether this stirring connects directly to molecular mixing at very fine scales, or whether there is an intermediate mechanism, in series with the eddy stirring, which accomplishes the transfer to mixing. Another question concerns the ultimate fate of the energy released from the mean state. Parameterization schemes essentially assume that this is dissipated adiabatically, but this may not be true. We return to both these issues later.

Another very serious question is whether the effect of mesoscale eddies can be represented as a purely local effect, with eddy fluxes just proportional to local mean gradients. This may be reasonable in some regions, such as the Antarctic Circumpolar Current, but inappropriate for non-zonal flows such as the Gulf Stream from which radiating Rossby waves and coherent rings may carry properties over large enough distances to invalidate any local mixing length approach. In such situations eddies may drive, rather than dissipate, the mean flow.
Ocean models may remain inadequate until mesoscale eddies are resolved explicitly. Moreover, apart from the difficulties associated with mesoscale eddies, high resolution also seems to be required to produce a sufficiently high effective Reynolds number for the correct representation of phenomena such as the separation, variability, and penetration of the Gulf Stream. Although results stabilize with increasing resolution, they remain sensitive to choices in forcing and viscosity parameterization, even at the finest grid spacing used today.

\section{General Approaches to Stirring}

Turbulent stirring leads to the distortion of a tracer field. The exact distortion is determined by the complete Lagrangian evolution of the flow field, but much can be inferred from the velocity gradient tensor. If the relative motion near a point is dominated by vorticity, then tracer gradients tend to align with the shear and not increase. If, on the other hand, the rate of strain dominates the relative motion, then a tracer tends to become elongated along the principal axis having the largest rate of strain and the tracer gradient grows exponentially. 


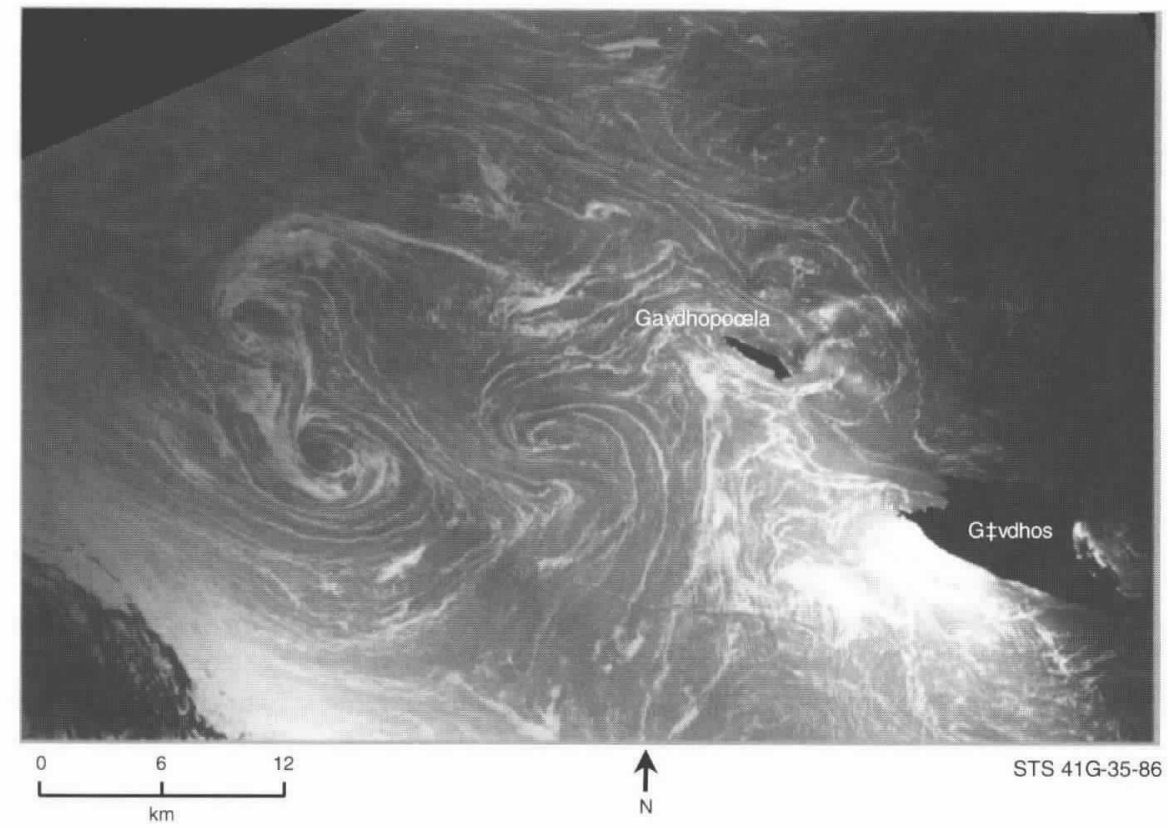

Figure 3. A pair of interconnected spirals in the Mediterranean Sea south of Crete. The vortex pair has a clearly visible stagnation point between the two spirals, the cores of which are aligned with the preconditioning wind field. Picture taken on October 7, 1984. (Courtesy W. Munk.)

Simulations of two-dimensional flows show additional phenomena such as barriers to mixing and areas of filament production. These barriers and areas can be identified when additional information is used. In one approach, the vorticity (from the velocity field) is combined with the rotation of the rate of strain tensor (from the acceleration field) into an effective rotation to characterize the flow field. Another approach uses concepts from dynamical systems theory. It identifies hyperbolic points in the flow field (characterized by strong deformations at stagnation points). These hyperbolic points are the origin of special material curves-or manifolds as they are called in the dynamical systems literature-that determine the barriers and pathways of advective transport. A blob of tracer can advect without much distortion, but then be ripped apart rather abruptly when it encounters these hyperbolic points in a flow. Realistic simulations of the surface currents in the Gulf of Mexico illustrate this process (Figure 2).

\section{Inertial Instability, Submesoscale Motions, and Vortical Motions}

Mesoscale motions may arise from baroclinic instability of large-scale flows in the ocean and may, in turn, be unstable to submesoscale eddies an order of magnitude smaller. Near the sea surface it seems that these eddies, often visible at the sea surface via beautiful spiral patterns (Figure 3), are mainly cyclonic, quite possibly because anticyclonic ones would break down rapidly via an inertial, or barotropic, instability. These small eddies may be a link in the pathway from stirring to mixing, but this has not been quantified. Similarly, in the ocean interior there seems to be a family of "vortical modes," of largely unknown scale range and intensity, which play a role in lateral stirring. They can be generated by frictional effects in flow past topographic features and, possibly, by the collapse and geostrophic adjustment of regions mixed by breaking internal waves.

A general question about the links between low frequency eddies and higher frequency motions concerns the extent to which the mesoscale or submesoscale eddies generate internal waves, particularly in situations where the eddies generate sharp fronts. Theoretically, the mesoscale and submesoscale eddies are assumed to satisfy some sort of balanced dynamics, such as geostrophy. These balanced motions are often called the "slow manifold." This manifold is different from the manifolds in the previous section. Balanced turbulent flows exhibit an upscale energy cascade and are very inefficient in dissipating energy. Internal gravity waves and other more nearly isotropic flows, on the other hand, exhibit a downscale cascade with effective dissipation. The structure of the equations governing balanced flows is such that they can be integrated forward in time only if certain conditions are met. Analysis of several classical instability problems shows that the fluid-dynamical transition occurs in the neighborhood of these integrability conditions. It is hypothesized that violation of these conditions characterizes, in general, the flow regimes where energy is transferred from balanced to unbalanced motions.

\section{The Interplay of Isopycnal and Diapycnal Processes}

When a scalar is stirred by high Reynolds number turbulence in homogeneous water, the eddy flux is mainly determined by the turbulent stirring and is largely independent of the small value of molecular 


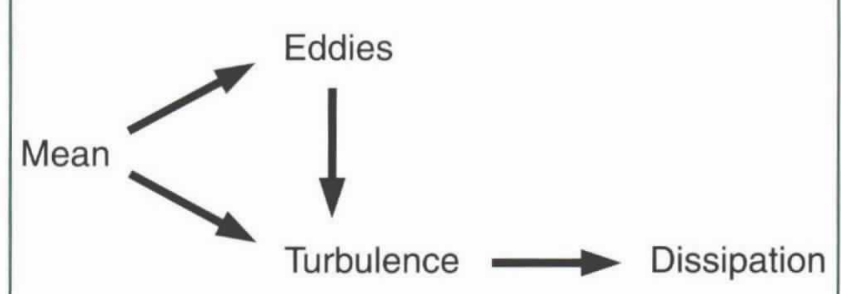

Figure 4. A simple schematic of the path of scalar concentration variance from mean to dissipation via eddies and turbulence. (Courtesy C. Garrett.)

diffusivity. Reducing the latter would merely lead to finer scales in the tracer field, without significantly affecting the turbulent transport. The situation is more subtle in the ocean because of the need to quantify both isopycnal and diapycnal transports. One issue, already raised, is the extent to which energy released by mesoscale eddies is dissipated in the ocean interior, perhaps by breaking internal waves which have drawn some of that energy from the eddies. This could partly tie diapycnal mixing rates to the lateral stirring rates.

Another issue concerns the ultimate mechanism for the destruction of fine-scale lateral gradients of temperature and salinity created by stirring on isopycnals. One possibility is that vertical shear of the stirring process leads to very strongly tilted frontal regions, so that pre-existing diapycnal mixing can remove the strong gradients. This could be described as a "passive" destruction of the strong lateral gradients. A more active process is possible: thermohaline fronts caused by lateral stirring on isopycnals could become unstable to a double-diffusive interleaving process. This would introduce further diapycnal mixing at the same time as it destroyed lateral gradients.

The relative importance of these scenarios has not been established. Preliminary estimates favor the latter process, the signature of which should be internal double-diffusive interleaving regions in the ocean. These are certainly known to occur, but a systematic catalog of their locations and characteristics has not been developed. If the process is important it may provide significant diapycnal mixing in regions with strong lateral gradients of potential temperature, and compensating salinity, on isopycnals. Moreover, the diapycnal mixing is upgradient for density, as is necessary in any double-diffusive process.

A significant sub-theme that emerged on this and other issues concerns the extent to which different stirring and mixing processes are in parallel or in series. If two processes are in parallel, their strengths may be compared to decide which is more important. If processes are in series, one might need to know which is the rate-controlling one. In the usual case of the stirring of a tracer in a homogeneous fluid, molecular mixing clearly operates in series, but with the ultimate

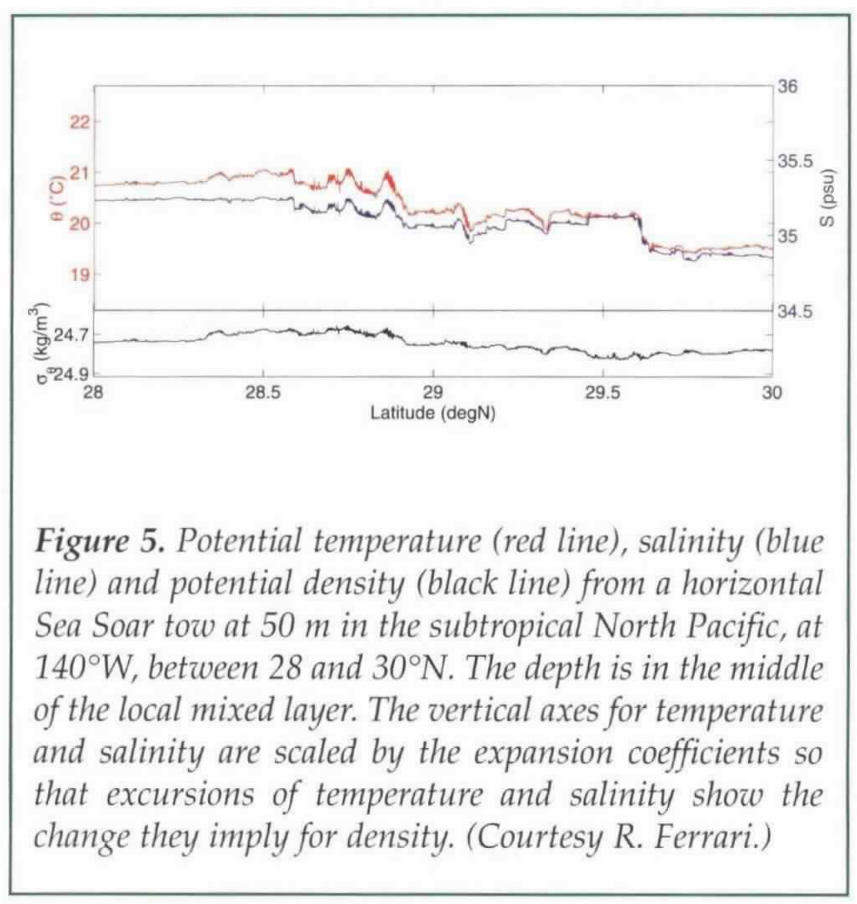

mixing rate being controlled by the turbulent stirring. In the ocean, where there might be some intermediate process between the stirring agents and the final molecular diffusion, the answer may be more complicated. For example, the flux of warm water across the Gulf Stream may be controlled by the amount of air-sea interaction that occurs in warm-core rings before they are reabsorbed by the stream; the flux is not necessarily controlled just by the ring formation rate. The general issue of series or parallel processes needs to be borne in mind in any attempt to focus on processes that are important, and may be aided by a triple decomposition into mean, eddies and turbulence (Figure 4).

\section{Processes in the Surface Mixed Layer}

Because of intense vertical mixing there, the surface mixed layer of the ocean provides many examples of the interplay of stirring and mixing processes. One such process relies on the fact that the density of seawater is determined by both temperature and salinity.

Typical of the surface mixed layer are compensated fronts that are warm and salty on one side and cold and fresh on the other side such that the density contrast is small. Figure 5 shows an example along a longitude line in the subtropical North Pacific. The existence of the fronts has been rationalized as follows (Young, 1994): Nonhomogeneous atmospheric forcing and entrainment of thermocline waters generate horizontal gradients in temperature, salinity, and density. Density fronts slump by the action of horizontal density-driven shear currents, which are eventually arrested by vertical mixing. This process can be thought of as shear dispersion. Compensated fronts do not experience such shear dispersion. The result is that density fronts diffuse whereas compensated fronts per- 
sist. The ubiquitous existence of compensated fronts suggests that horizontal mixing in the surface mixed layer is very much larger for density than for "spice," the combination of temperature and salinity that is "orthogonal" to density. Indeed, eddy-resolving models that diffuse temperature and salinity and hence density and spice with the same coefficient are unable to reproduce the observed tendency towards thermohaline compensation.

Air-sea fluxes and the behavior of the surface mixed layer are also involved in a thermodynamic approach to water-mass formation. Basically, water must flow across isopycnals to balance surface buoyancy loss or gain, and the convergence of this volume flux provides an estimate of the subduction rate. This rate may be compared with a dynamically based prediction from Ekman convergence. Both the thermodynamic and dynamic approaches, however, require allowance for the role of eddies and diurnal and seasonal cycling of the mixed layer depth. The problem is thus complicated by the interplay of various stirring and mixing processes, which need to be understood.

\section{The Stirring and Mixing of Non- Conservative Tracers}

Remote sensing of the sea surface from space has made us aware of beautiful and complex patterns in biologically active variables such as chlorophyll (Figure 6). One question is whether the observed patchiness is a consequence of biological processes or merely of the passive stirring of large-scale gradients. Some clues come from comparing the shapes of the wavenumber spectra of biologically active and passive scalars, such as chlorophyll and temperature respectively. If the former shows comparatively more variance at small scales, this is evidence for the importance of biological growth and decay processes.

Accounting for such patchiness in a model is particularly difficult if the non-conservative processes are nonlinear. In that case it is clearly inadequate to proceed with a model that deals only with an ensemble average variable; a Lagrangian approach which follows the development of individual patches is necessary. A very basic model of this kind is the "Brownian bug model." It describes an ensemble of bugs that perform random walks and experience random birth and death processes along the way. Mathematically, this is a "superprocess." A homogeneous initial distribution spontaneously develops patches and voids.

Examining data for evidence of patchiness or intermittency can be aided by examination of high order structure functions. For a variable $C(x, t)$ the $q$ th order structure function is defined as $\left\langle(\delta C(1))^{9}\right\rangle$, where $\delta C(l)$ is the difference in $C$ over a separation $l$ and \langle\rangle denotes an ensemble average. The structure function exponent $\zeta(q)$, defined by fitting a power law $l^{(\xi)}$ to the structure function, characterizes the degree of intermittency for multifractal processes. It is a linear function of $q$ for non-intermittent processes and becomes a nonlinear

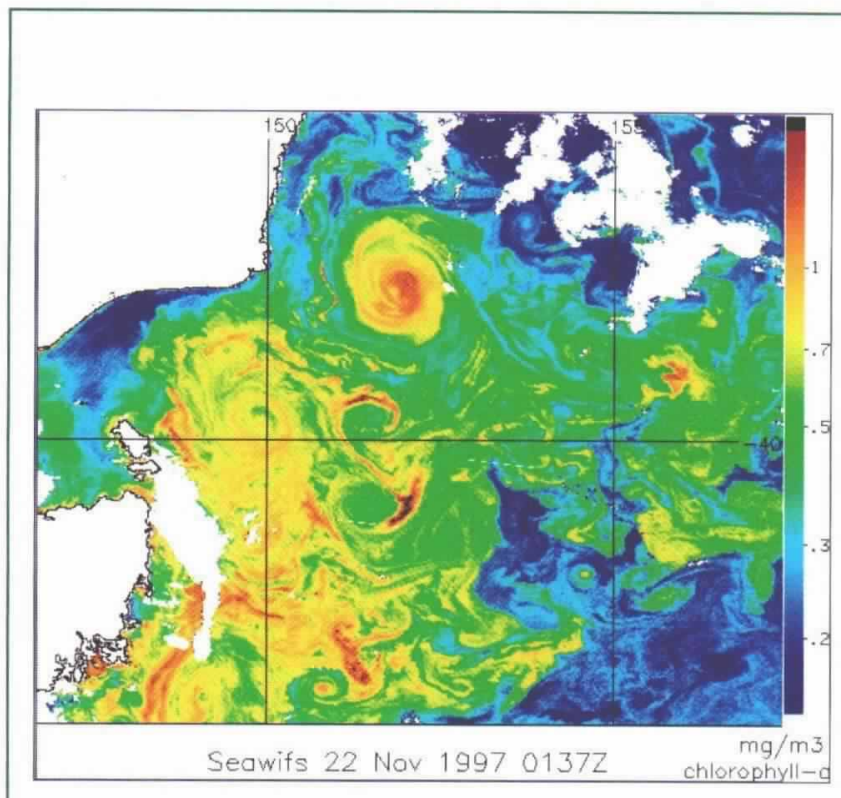

Figure 6. Chlorophyll concentration in the Tasmanian Sea as observed by SeaWiFS. (Courtesy B.L. Hua.)

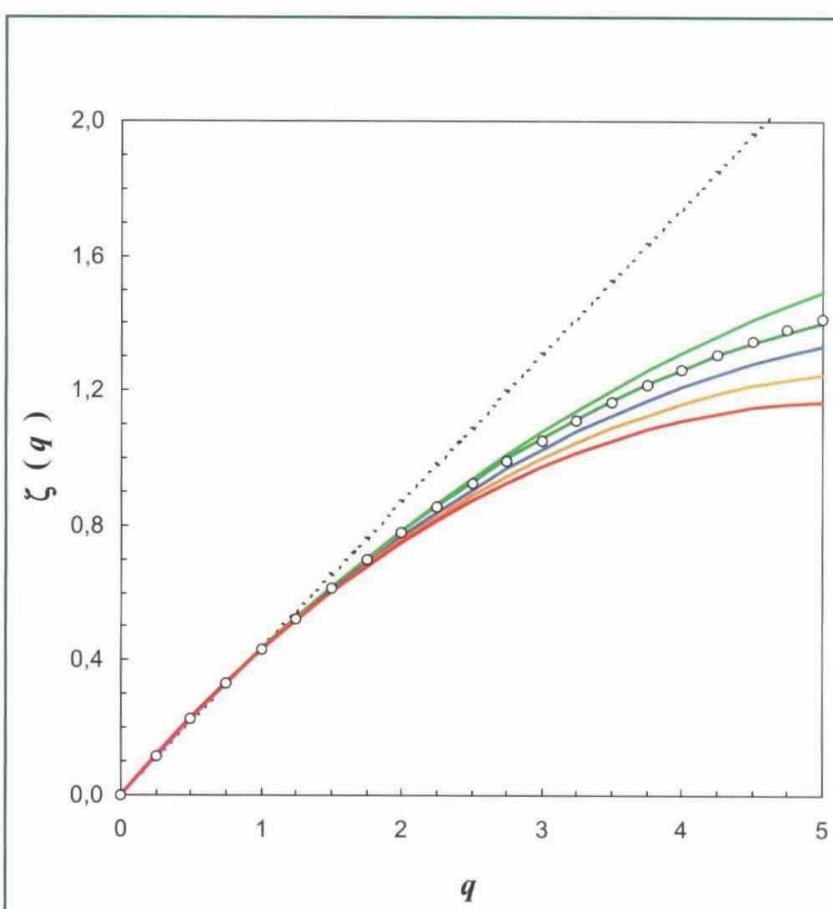

Figure 7. Empirical values of the structure function exponent $\zeta(\mathrm{q})$ for temperature (circles) and in vivo fluorescence for different values of the turbulent dissipation rate $\varepsilon$ (red $\varepsilon=10^{-6} \mathrm{~m}^{2} \mathrm{~s}^{-3}$; orange $\varepsilon=5 \times 10^{-6} \mathrm{~m}^{2} \mathrm{~s}^{-3} ;$ blue $\varepsilon$ $=10^{-5} \mathrm{~m}^{2} \mathrm{~s}^{-3}$; dark green $\varepsilon=5 \times 10^{-5} \mathrm{~m}^{2} \mathrm{~s}^{-3}$; light green $\varepsilon=$ $10^{-4} \mathrm{~m}^{2} \mathrm{~s}^{-3}$ ). The linear curve (dotted line) is expected for non-intermittent turbulence. The data were collected in the eastern English Channel. (Courtesy L. Seuront.) 
concave function for intermittent processes. Calculation of this exponent from oceanic observations suggests that, in the scale range of nearly isotropic turbulence, passively stirred scalars such as temperature are more intermittent than the advecting velocity field. This is a result also obtained in numerical simulations of 3D homogeneous turbulence (e.g., Jiménez, 2000). Biologically active tracers show intermittency beyond that associated with turbulent stirring. The excess increases with decreasing turbulence intensity (Figure 7). These observational results need to be corroborated and understood. Further to this, the possibility of a relationship between biological and physical variables can be studied through examination of the behavior of joint structure functions, though caution is required to avoid overinterpretation of artifacts introduced by particular sampling schemes or noise.

\section{Mixing Efficiency}

Much of the diapycnal mixing in the ocean is associated with the shear instability of internal waves. The subsequent overturning and stirring leads to fine scale patterns of density which then disappear through the action of molecular thermal diffusivity or the molecular diffusion of salt.

The turbulent eddies are being dissipated by molecular viscosity at the same time as they are working against buoyancy forces; a major question concerns the relative fractions of turbulent kinetic energy lost to dissipation at a rate $\varepsilon$ and converted to mean potential energy at a rate $K_{i} N^{2}$, where $K_{\text {r }}$ is the eddy diffusivity and $N$ the buoyancy frequency. The ratio $\Gamma=K_{i} N^{2} / \varepsilon$ is generally thought to be about 0.2 , largely based on calibration against other methods of estimating $K$. There is no reason to suppose that $\Gamma$ is a universal constant, however, but it has not been established what environmental parameters might determine its value.

One suggestion is that a key parameter is the "turbulent Reynolds number" $R e^{\prime}=\varepsilon / \mathrm{vN}^{2}$. This is a measure of the strength of the turbulence; it can, for example, be written as $\left(L / L_{t}\right)^{4.3}$, where the Ozmidov scale $L_{t}=\left(\varepsilon / N^{3}\right)^{1.2}$ is a measure of the scale of overturns, and $L_{k}=\left(v^{2} / \varepsilon\right)^{1.4}$ is the Kolmogoror scale at which viscosity significantly damps the turbulent eddies.

Some evidence reported at the meeting, based largely on a laboratory experiment but with some field support, suggests that $I$ ' is less than the value of about 0.2 quoted above if the turbulence is weak with a turbulent Reynolds number less than about 100. This is not surprising: weak turbulence dies away before it has accomplished much buoyancy flux. What was a surprise was a claim that $\Gamma$ also falls off again for values of $R e^{2}$, greater than about 1,000. This dependence is hard to accept for a situation with overturning scales much larger than the viscous cut-off scale; it may transpire that $R e^{\prime}$ in these experiments was actually a proxy for an input variable that does not involve the viscosity $z$.

One variable that might lead to reduced $I$ in the ocean is the duration of high shear compared with the buoyancy time $\mathrm{N}^{-1}$. A long-lived mixing event might produce all its buoyancy flux at first and subsequently stir water that is already mixed, dissipating energy but with little further buoyancy flux. More study is required, with high resolution 3D numerical simulations of breaking internal wave fields becoming increasingly feasible and informative.

The question of mixing efficiency is important in any assessment of the amount of mixing that might be associated with, for example, the energy loss from the barotropic tide to internal tides. There are also implications for the circulation that is diagnosed from measured dissipation rates $\varepsilon$ and the observed mean density structure. The mean diapycnal velocity $u$, may be obtained from the mean buoyancy equation (e.g., St. Laurent et al., 2001)

$$
w \cdot N^{\prime}=\partial(\Gamma \varepsilon) / \partial z
$$

so that the value of $w$. can depend on gradients of $\Gamma$. Vortex stretching given by $f \partial u+\partial z$ then affects the mean flow along isopycnals.

\section{Differential Mixing}

The rapid decay of turbulent kinetic energy at small values of the turbulent Reynolds number $R e_{l}=$ $\varepsilon /{ }^{2} N^{2}$ can also lead to less turbulent transport of salt than heat, given the very small molecular diffusivity of the former. Although the effect is clearly established in $2 \mathrm{D}$ and $3 \mathrm{D}$ numerical simulations and in laboratory experiments that mimic the breaking of internal waves in a stratified ocean, it is somewhat counterintuitive at first sight. If the turbulent fluxes were downgradient at all scales then the more diffusive tracer would lose its higher wavenumber fluctuations and would experience less turbulent transport. It seems, however, that the fluxes are upgradient at high wavenumbers, as a simple consequence of the secondary instability and collapse of an overturning billow. The more diffusive tracer thus loses some upgradient flux and therefore experiences more downgradient transport.

The importance of differential mixing for the ocean is not yet known. It may turn out that in regions where mixing matters, it is at high enough $R e_{\text {: }}$ that the eddy diffusivities of heat and salt are the same. This is particularly likely to be true given the intermittent nature of mixing in the ocean compared with the rather steady stirring in the numerical experiments; $R e_{t}$ based on $\varepsilon$ during a mixing event in the ocean may be much larger than that based on the average $\varepsilon$.

Different rates of mixing for heat and salt are, however, firmly established for regions of the ocean where double-diffusive processes are significant. These 
regions include places, such as the C-SALT region near Barbados (Schmitt, 1994), where the vertical profiles of temperature and salinity are nearly compensating in density, and places such as the Arctic Ocean where internal wave activity is low. Even in these regions there are significant puzzles, partly because laboratory results seem not to be readily applicable, and partly because we lack adequate theoretical guidance. One obvious problem is to explain the step size in thermohaline staircases. In the diffusive regime (with warm salty water below cooler fresher water), some useful predictions come from a local water column model, whereas in the salt fingering regime (with the opposite stratification) it seems that the step size might be set by lateral intrusive processes. The issue is certainly not fully resolved, and the interfaces in the fingering regime also seem more complex in the ocean than in the laboratory.

Importantly, many regions of the ocean which might support double-diffusive processes seem to have these disrupted by things like the shear of inter-nal waves, with the breaking of these waves then dominating diapycnal transports. Even these regions can show some evidence for double diffusive transports, however, so that the phenomena warrant continued attention.

\section{Summary}

There are typically seven orders of magnitude between the smallest scales being resolved by numerical models of ocean circulation and the smallest scales of variability. The meeting was therefore faced with a vast range of scales, phenomena and issues. It focused on topics where tangible progress had recently been made and where underlying issues had been brought into sharper relief. It is not really clear what issues have the highest priority for future research, but these are the issues that came up repeatedly at the meeting:

1. What are the constraints on eddy parameterization and how should they be implemented?

2. What nonlocal effects matter and how can they be parameterized?

3. How uniform are stirring and mixing processes? Are there any "hotspots," particularly in association with topographic features?

4. What are the effects of diapycnal boundary processes on eddy parameterization?

5. What is the parameter dependence of the mixing efficiency or flux Richardson number?

6. How does energy leak from the slow manifold?

7. What aspects of stirring and mixing affect biological processes? What do patterns of biological tracers tell us about fluid dynamics?

8. Under what circumstances do we need different diffusivities for different tracers?

9. What are the effects of adiabatic stirring on diapycnal mixing?
10. Do subgridscale processes provide stochastic forcing?

The last question addresses a very fundamental aspect of the Reynolds decomposition. A second fundamental aspect is that the simple Reynolds decomposition ideally requires a spectral gap, in time or space, separating the unresolved processes to be parameterized from the slowly changing "mean."

Stirring and mixing in a stratified ocean is the physics that needs to be parameterized in ocean circulation models. There is a need to understand these processes and to parameterize them in a form that can be implemented in models for the large-scale behavior of the ocean. These models should be used in sensitivity tests to determine which parameterizations need improvement. These are the challenges ahead of us.

\section{Acknowledgments}

We thank the participants of the workshop for their input into this report and for their permission to quote unpublished material. Copies of the proceedings are available from Peter Müller, University of Hawaii, School of Ocean and Earth Science and Technology, Department of Oceanography, 1000 Pope Road, Honolulu, Hawaii, 96822, and can also be viewed at http:/ / www.soest.hawaii.edu/PubServices/AhaHuli koa.html. The twelfth 'Aha Huliko'a Hawaiian Winter Workshop was supported by the Department of the Navy grant number N00014-00-1-0168, issued by the Office of Naval Research, and by the Joint Institute for Marine and Atmospheric Research.

\section{References}

Eckart, C., 1948: An analysis of the stirring and mixing processes in incompressible fluids. J. Mar. Res., 7, 265-275.

Gent, P.R. and J.C. McWilliams, 1990: Isopycnal mixing in ocean circulation models. J. Phys. Oceanogr., 20, 150-155.

Jiménez, J., 2000: Turbulence. In: Developments in Fluid Mechanics. G.K. Batchelor, K. Moffatt and G. Worster, eds., Cambridge University Press, 231-288.

St. Laurent, L., J. Toole and R. Schmitt, 2001: Buoyancy forcing by turbulence above rough topography in the abyssal Brazil Basin. J. Phys. Oceanogr., 31, 3476-3495.

Schmitt, R.W., 1994: Double diffusion in oceanography. Ann. Rev. Fluid Mech., 26, 255-285.

Welander, P., 1955: Studies of the general development of motion in a two-dimensional, ideal fluid. Te'llus, 7, 141-156.

Young, W.R., 1994: The subinertial mixed layer approximation. J. Phys. Oceanogr., 24, 1812-1826. 